\title{
Diffuse Parenchymal Neurocysticercosis: A Case Report and Review of Literature
}

\author{
Hanuman Prasad Prajapati ${ }^{1}$ \\ ${ }^{1}$ Department of Neurosurgery, Uttar Pradesh University of Medical \\ Sciences (UPUMS), Saifai, Etawah, Uttar Pradesh, India
}

\begin{abstract}
Address for correspondence Hanuman Prasad Prajapati, MCh Neurosurgery, Department of Neurosurgery, Uttar Pradesh University of Medical Sciences (UPUMS), Saifai, Etawah 206130, Uttar Pradesh, India (e-mail: pushpa84.dhp@gmail.com).
\end{abstract}
Abstract
Keywords
- neurocysticercosis
- diffuse
- radiology
- diagnostic criteria
- management

Neurocysticercosis is the commonest parasitic disease of the central nervous system and the leading cause of seizure in the developing world. Human cysticercosis is caused by the dissemination of the embryo of Taenia solium in the intestine via the hepatoportal system to the tissues and organs of the body. The organs most commonly affected are the subcutaneous tissues, skeletal muscles, lung, brain, eye, liver, and occasionally the heart, thyroid, and pancreas. Widespread dissemination of the cysticerci can result in the involvement of almost any organ in the body. We report a case of 60 years old male presented with head injury due to fall, which was diagnosed as diffuse parenchymal neurocysticercosis on computed tomography scan of brain. We discuss the radiological feature, diagnostic criteria, management plan, and literature review of such reported cases.

\section{Introduction}

Neurocysticercosis (NCC) is the most common parasitic disease of the central nervous system. According to World Health Organization, NCC is the most common preventable cause of epilepsy in the developing world, with an estimated 2 million people having epilepsy caused by Taenia solium infection. ${ }^{1}$ Symptomatic NCC accounts for approximately one-third of seizure disorders. ${ }^{2}$ Clinically, it can manifest as epilepsy, intracranial hypertension, focal neurological signs, cerebellar ataxia, cognitive decline, symptoms of hydrocephalus and psychiatric disorders. The clinical manifestations vary with the number, size, location and stage of cysticerci as well as the intensity of the host immune response. ${ }^{3}$

NCC remains a major public health problem in most of the world. Millions of individuals are estimated to be infected; many of whom become symptomatic at some point in their lives. ${ }^{2}$ Extraparenchymal NCC is still associated with high mortality rates, mainly due to intracranial hypertension, whereas mortality in parenchymal NCC is limited to epilepsyrelated deaths or a high burden of cysts. ${ }^{4}$

\section{Case Report}

A 60-years-old male patient presented to us with alleged history of fall on the ground following seizure 1 day back. Patient was also having past history of forgetfullness, abnormal behavior, and complex partial seizure since 2015. There was no history of cerebrospinal fluid (CSF) rhinorrhea/otorrhea, diabetes mellitus, hypertension, and fever. On examination, patient was conscious and oriented. His mini mental status examination was $26 / 30$. His cranial nerve, motor, sensory, and cerebellum system examination was in the normal limit.

Patient previously consulted to a neurologist and was evaluated with computed tomography (CT) and magnetic resonance imaging (MRI) brain, and diagnosed as a case of diffuse NCC ( - Fig. 2A, 2B). As per treatment record, patient had taken antihelminthic and anticonvulsant treatment. Recent noncontrast computed tomography brain was suggestive of diffuse intraparenchymal NCC with multiple droplets of pneumocephalus ( - Fig. 1A, 1B). Patient was managed conservatively and discharged after 3days on anticonvulsant treatment.

\section{Discussion}

NCC is the most common helminthic infection of the nervous system and is a major cause of acquired epilepsy worldwide. It is endemic in Latin America, South East Asia, India, Nepal, China, Africa, and many other developing countries. With increasing globalization and international travel, NCC is now being reported from many developed countries. ${ }^{5}$
License terms

(®) $\Theta \circledast$ 


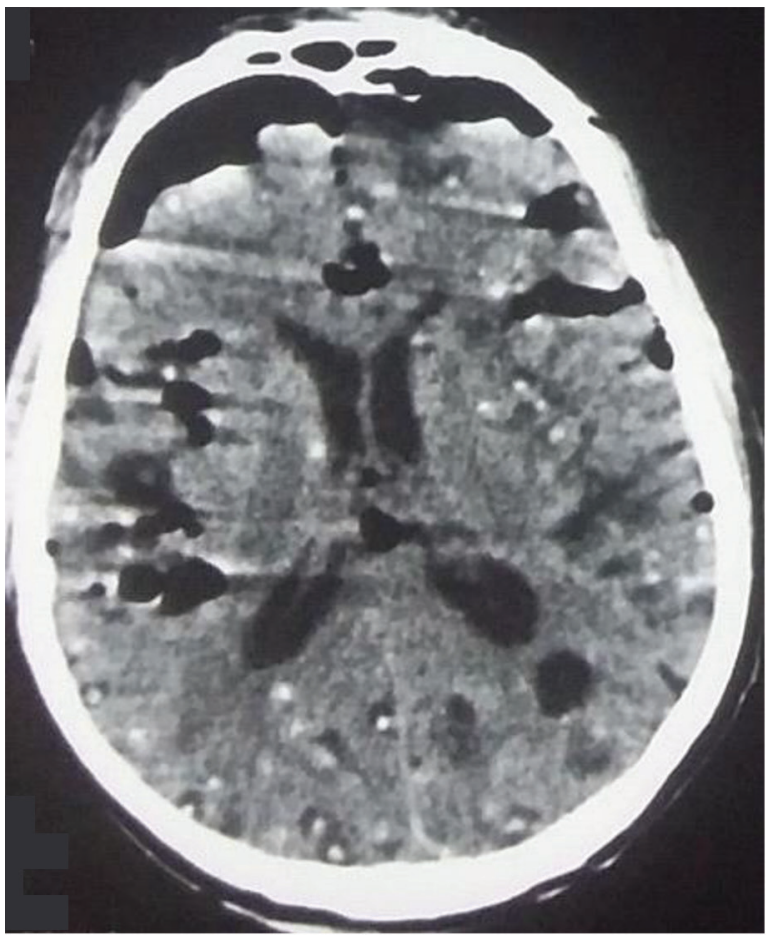

A

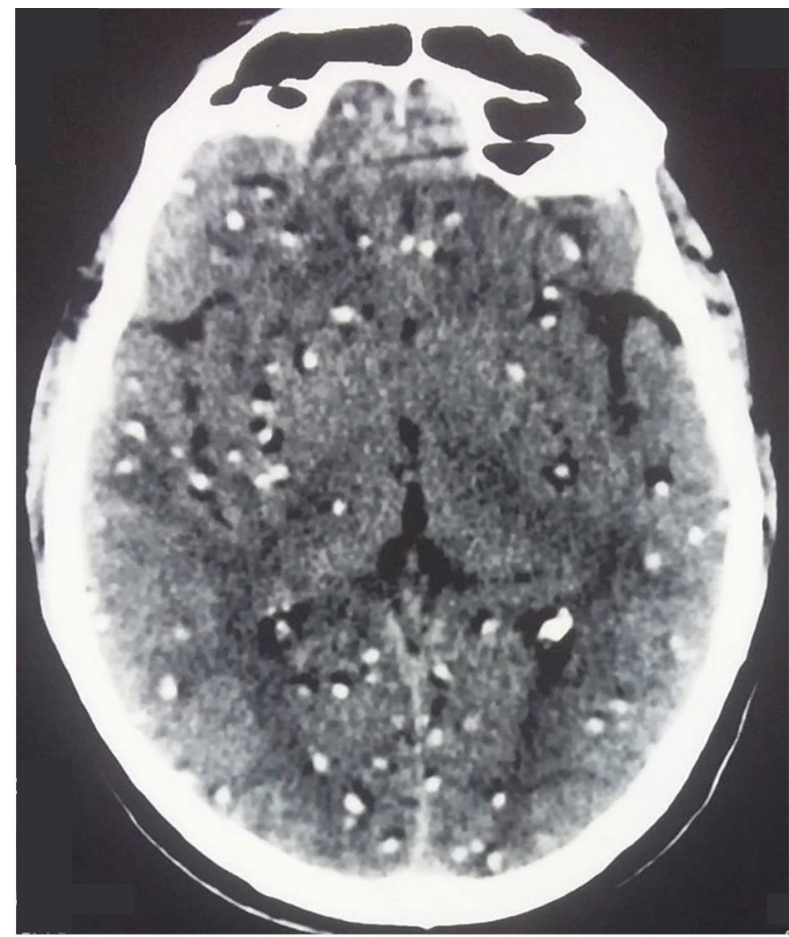

B

Fig. 1 (A) Plain computed tomography scan of brain showing multiple scattered calcified neurocysticercosis throughout the brain parenchyma with multiple pneumocephalus. (B) Plain CT scan of brain showing multiple scattered calcified neurocysticercosis throughout the brain parenchyma.

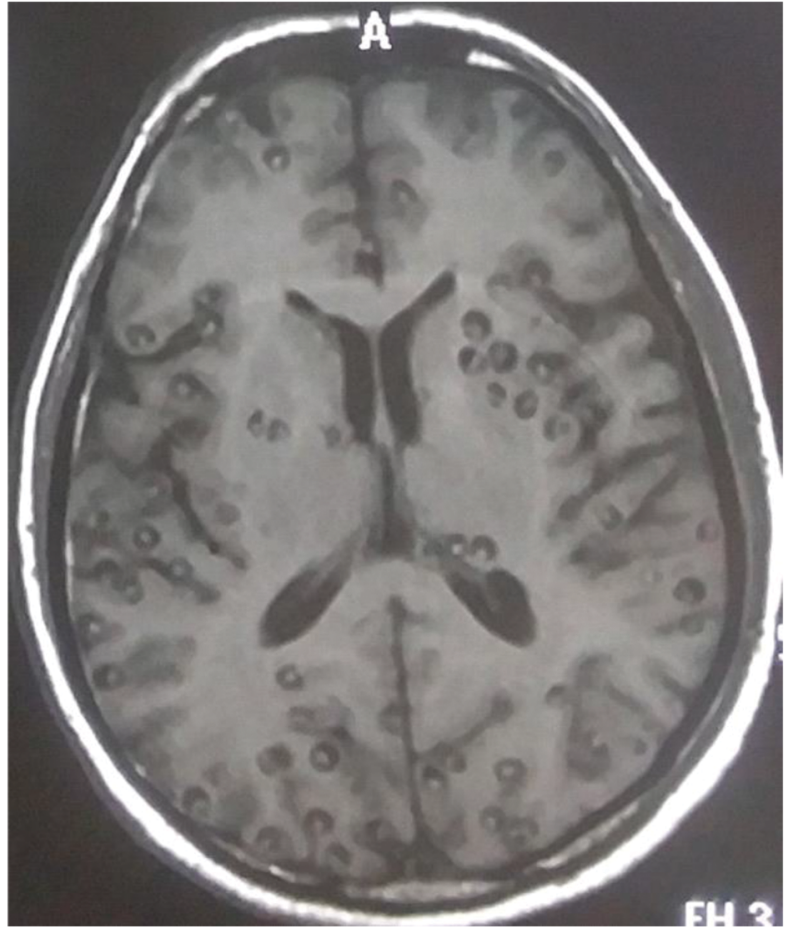

A

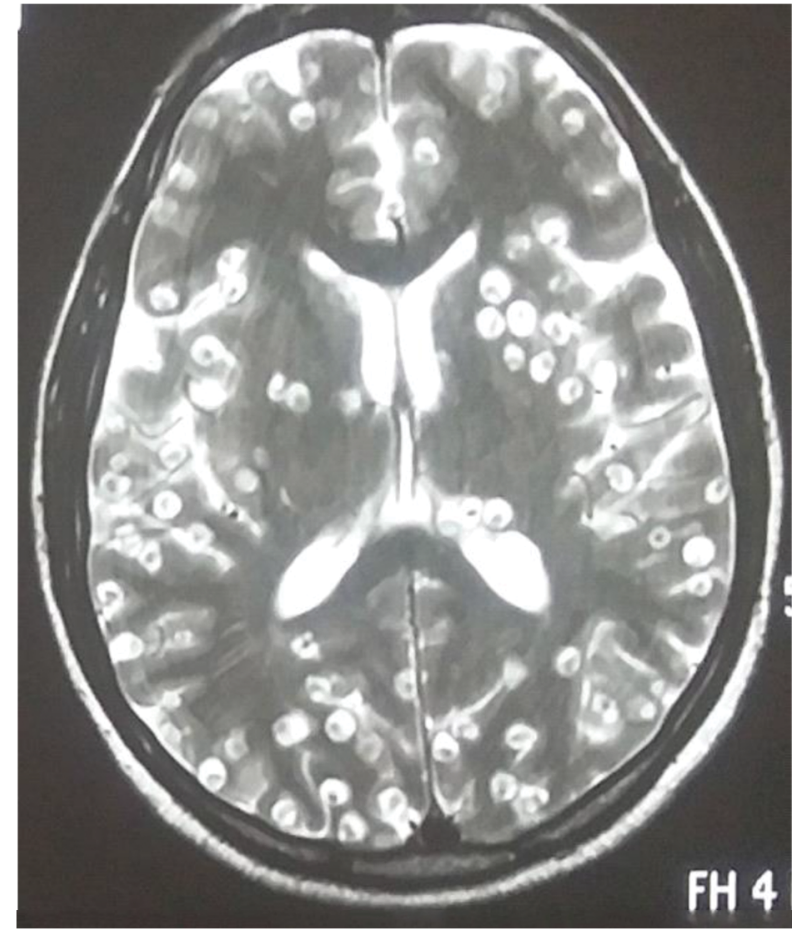

B

Fig. 2 (A) Magnetic resonance imaging brain T1-weighted axial image showing numerous cysts with hyperintense eccentric lesion in the brain parenchyma. (B) Magnetic resonance imaging brain T2-weighted axial image showing numerous hyperintense cyst with hypointense eccentric lesion in the brain parenchyma. 


\section{Neuroimaging}

CT scans and MRI are useful in anatomical localization of the cyst. MRI is more sensitive than CT, as it not only identifies scolex and live cyst in cisternal spaces and ventricles but also identifies the response to treatment. ${ }^{6}$

\section{Staging}

According to Escobar classification, NCC is categorized into four stages. ${ }^{7}$

Stage 1: Vesicular stage: It is the active or viable stage. CT scan shows round, hypodense areas of varying in sizes and numbers, with hyperdense eccentric mural nodule and no perilesional edema. There is no enhancement on contrast study. On MRI, it appears as CSF-like intensity signal on all sequences with hyperintense mural nodule ( - Fig. 2A, B).

Stage 2: Colloidal vesicular stage: It is the transitional stage. The cysticercus loses the ability to control the host immune response and the cyst wall becomes infiltrated and is surrounded by predominantly mononuclear cells. On CT and MRI, it appears as ring enhancing lesions with perilesional edema.

Stage 3: Granular nodular stage: It is also the transitional stage in which host immune response further progresses with collapse of the cyst cavity. On CT, it appears as small, hyperdense, rounded, nodular lesion surrounded by edema. On MRI, it appears as signal void in all the sequences with perilesional edema.

Stage 4: Calcified nodular stage: It is the inactive stage formed as a result of dystrophic calcification of the necrotic larva. It is seen as calcified nodule with no perilesional edema on CT scan ( - Fig. 1A, B). On MRI, it is seen as signal void in all the sequences with no perilesional edema.

\section{Diagnosis}

\section{Radiological Diagnosis}

Neuroimaging is the method of choice for the diagnosis of NCC. It includes cranial CT and/or MRI, which should be combined with serological tests. In neuroimaging, only a lesion showing the scolex (head of the parasite) is classified as a definite NCC lesion and considered pathognomonic. ${ }^{9}$ Active NCC is defined as the presence of any cystic lesions (with or without scolex) or lesions with ring enhancement. Nodular enhancing lesions are often termed transitional. In contrast, parenchymal calcifications are classified as inactive. Lesions highly suggestive of NCC are of the active, transitional, and inactive type. ${ }^{10}$

\section{Immunological Diagnosis}

Serological tests for detecting antibodies against cysticercosis are used to confirm the diagnosis. ${ }^{11}$ The best documented serological test is the enzyme-linked immune electrotransfer blot (EITB) assay. EITB specificity approaches $100 \%$ with a sensitivity around $98 \%$ for patients with two or more live parasites in the nervous system.

Thus, people with more than one viable cyst or subarachnoid disease at the time of testing will have a positive serology. Thus, EITB is frequently falsely negative in patients with a single intracranial cysticercus (including the Indian variant of solitary cerebral cysticercus granuloma) or in those with calcified cysticerci. ${ }^{8}$ Therefore, a negative test cannot exclude NCC.

\section{X-Ray}

Plain radiography can be useful for the evaluation of extraneural cysticercosis such as calcified lesions ("cigar-shaped calcifications") in muscle or subcutaneous tissue. However, a CT scan is more sensitive for assessing these findings. ${ }^{12}$

\section{Management}

The treatment of NCC depends upon the viability of the cyst and its complications. Steroids, antihelminthics, and antiepileptics are used for treatment. The cysts in the active and transitional stage are treated with steroids. ${ }^{1}$

In patients with raised intracranial tension, surgical removal of cysts and ventriculoperitoneal shunting can alleviate the symptoms. Pharmacological management with cysticidal drugs praziquantel ( $10-15 \mathrm{mg} / \mathrm{kg} /$ day for $6-21$ days) and albendazole ( $15 \mathrm{mg} / \mathrm{kg} /$ day for 30 days) is indicated, as they help by reducing the parasite burden. Pharmacological treatment may be associated with severe reactions, which may result in the enlargement of the cysts, massive release of antigens causing local tissue swelling, and a generalized anaphylactic reaction. Priming with corticosteroids before starting the cysticidal drug decreases the incidence of such complications. ${ }^{7}$ There is no role for cysticidal drug in inactive NCC (stage 4), as the parasites are dead and given only symptomatic treatment. ${ }^{13}$

Though disseminated cysticercosis has been reported as early as 1912 by the British Army medical officers stationed in India, only 116 cases have been reported till date worldwide, the majority being from India. ${ }^{14}$ Definition of disseminated cysticercosis is still not known. We propose that the diagnosis of disseminated cysticercosis can be considered to be confirmed if there are multiple vesicular cystic lesions present in the brain and cysts are demonstrated in at least two other body parts. ${ }^{15}$ Why is disseminated cysticercosis common in India? Latest PubMed data revealed that a majority of reports related to disseminated cysticercosis are from India. Both environmental (poor hygienic and sanitary conditions) and genetic factors are possibly responsible for this occurrence. What is the natural course of disseminated cysticercosis? The exact course in disseminated cysticercosis is not precisely known. Individual cysts either get inflamed and/or get calcified and remain in the brain, unchanged even after several years. 


\section{Conclusion}

NCC is the commonest parasitic infection of brain and a leading cause of seizure in the developing world. CT and MRI are the best imaging modalities for diagnosing, staging, and recurrence of NCC. Response to treatment can also be evaluated on neuroimaging.

\section{Funding}

None.

\section{Conflict of Interest}

None declared.

\section{References}

1 Singh G, Burneo JG, Sander JW. From seizures to epilepsy and its substrates: neurocysticercosis. Epilepsia 2013;54(5): 783-792

2 Del Brutto OH, Garcia HH. Neurocysticercosis. Handb Clin Neurol 2013;114:313-325

3 Takayanagui OM, Odashima NS. Clinical aspects of neurocysticercosis. Parasitol Int 2006;55(Suppl):S111-S115

4 Fleury A, Carrillo-Mezo R, Flisser A, Sciutto E, Corona T. Subarachnoid basal neurocysticercosis: a focus on the most severe form of the disease. Expert Rev Anti Infect Ther 2011;9(1):123-133

5 Singhi P. Neurocysticercosis. Ther Adv Neurol Disorder 2011;4(2):67-81
6 Bhalla A, Sood A, Sachdev A, Varma V. Disseminated cysticercosis: a case report and review of the literature. J Med Case Reports 2008;2:137

7 Escobar A, The pathology of neurocysticercosis of the central nervous system. In: Palacios E, Rodriguez- Carbajal J, Taveras JM, eds. Cysticercosis of the Central Nervous System. Springfield, IL: Charles C Thomas; 1983 27-54

8 Garcia HH, Nash TE, Del Brutto OH. Clinical symptoms, diagnosis, and treatment of neurocysticercosis. Lancet Neurol 2014;13(12):1202-1215

9 Pal DK, Carpio A, Sander JW. Neurocysticercosis and epilepsy in developing countries. J Neurol Neurosurg Psychiatry 2000;68(2):137-143

10 Nash TE, Singh G, White AC, et al. Treatment of neurocysticercosis: current status and future research needs. Neurology 2006;67(7):1120-1127

11 Sardana V, Ojha P, Sharma D, Sharma SK, Saxena S, Rai NN. Disseminated cysticercosis. Neurol India 2016;64(5): 1058-1060

12 Bustos JA, Garcia HH, Dorregaray R, et al; Cysticercosis Working Group in Peru. Detection of muscle calcifications by thigh CT scan in neurocysticercosis patients. Trans R Soc Trop Med Hyg 2005;99(10):775-779

13 Garg RK. Drug treatment of neurocysticercosis. Natl Med J India 1997;10(4):173-177

14 Muthukumar N. Commentary: neurocysticercosis: evolution of our understanding. Neurol India 2017;65(4):885-887

15 Garg RK. Diagnostic criteria for neurocysticercosis: some modifications are needed for Indian patients. Neurol India 2004;52(2):171-177 